\title{
Nanocompósitos de Poliamida 6/Argila Organofílica: Efeito do Peso Molecular da Matriz na Estrutura e Propriedades Mecânicas e Termomecânicas
}

\author{
Renê A. da Paz, Amanda M. D. Leite \\ Ciência e Engenharia de Materiais, UFCG \\ Edcleide M. Araújo, Tomas J. A. Melo \\ Departamento de Engenharia de Materiais, UFCG \\ Renata Barbosa \\ Engenharia de Processos, UFCG \\ Edson N. Ito \\ Embrapa, São Carlos/SP
}

\begin{abstract}
Resumo: Nanocompósitos de poliamida 6/argila organofílica foram preparados pelo método de intercalação por fusão. A argila foi tratada com o sal quaternário de amônio (Genamin) visando-se a obtenção da argila organofílica (OMMT). Ela foi caracterizada por fluorescência de raio X (FRX) e difração de raio X (DRX) e, os resultados dessas análises mostraram a incorporação do sal entre as camadas da argila, tornando-a organofílica. Os nanocompósitos foram obtidos em extrusora de rosca dupla contra-rotacional, com 3\% em peso de argila e, foram caracterizados por difração de raio X (DRX), microscopia eletrônica de transmissão (MET), caracterização mecânica sob tração, análise térmica dinâmico-mecânica (DMTA) e temperatura de deflexão térmica (HDT). Os resultados de DRX e MET mostraram estrutura esfoliada e/ou parcialmente esfoliada. As propriedades mecânicas sob tração dos nanocompósitos foram superiores às da poliamida $6 \mathrm{e}$, a análise por DMTA e HDT mostrou que a incorporação da argila organofílica na matriz polimérica, aumentou a rigidez (módulo de armazenamento) e a HDT do sistema, confirmando o efeito reforçante da argila no polímero.
\end{abstract}

Palavras-chave: Nanocompósitos, poliamida 6, argila organofílica.

Polyamide 6/Organoclay Nanocomposites: Effect of Matrix Molecular Weight on Structure and Mechanical and ThermalMechanical Properties

Abstract: Polyamide 6/organoclay nanocomposites were prepared by the melt intercalation technique. The clay was treated with a quaternary ammonium salt (Genamin) to obtain the organoclay (OMMT), being characterized by $\mathrm{X}$ ray fluorescence (XRF) and X ray diffraction (XRD). The results confirmed the incorporation of the salt into the clay structure, i.e. the organophilization. The nanocomposites were obtained with a counter-rotational twin screw extruder with $3 \mathrm{wt} . \%$ of clay and characterized by XRD, transmission electron microscopy (TEM), tensile testing, dynamical-mechanical thermal analysis (DMTA) and heat deflection temperature (HDT). The XRD and TEM results showed exfoliated and/or partially exfoliated structures. The tensile properties were superior to those of the pure polyamide 6.The analysis with DMTA and HDT also showed that the incorporation of the organoclay into the polymer matrix increased the rigidity (storage modulus) of the system, confirming the reinforcing effect of the clay on the polymer.

Keywords: Nanocomposites, polyamide 6, organoclay.

\section{Introdução}

A partir da década de 60, começou-se a se reportar o desenvolvimento dos primeiros nanocompósitos de polímero/argila quando Blumstein citado por Leite ${ }^{[1]}$ demonstrou, por meio da técnica de polimerização de monômeros vinílicos, a intercalação de moléculas poliméricas entre as lamelas da montmorilonita. A partir daí, outros mé- todos têm sido desenvolvidos para preparação de nanocompósitos polímero/argila ${ }^{[2]}$. Atualmente, vem sendo dada muita atenção aos nanocompósitos poliméricos, especialmente aos nanocompósitos desenvolvidos com silicatos em camada, devido à grande necessidade de materiais modernos de engenharia e ao fato dos polímeros puros não apresentarem as propriedades necessárias para certas aplicações ${ }^{[3]}$. 
Nanocompósitos polímero/silicato são materiais em que partículas de dimensões nanométricas estão dispersas em uma matriz polimérica. Assim como os chamados compósitos poliméricos, os nanocompósitos utilizam cargas para reforçar as propriedades dos polímeros. Porém, as propriedades obtidas para os nanocompósitos são únicas, pois estes utilizam baixos teores de silicato (1-5\% em peso) e apresentam, teoricamente, boas propriedades quando comparadas aos compósitos convencionais, tais como: melhores propriedades mecânicas, térmicas, inflamabilidade e químicas, menor permeabilidade a gás e maior estabilidade dimensional. Para que isto ocorra, é necessário que a argila esteja uniformemente dispersa no polímero e que haja interação interfacial entre a matriz polimérica e o silicato. Portanto, tratamentos superficiais das argilas com sais orgânicos são indispensáveis para que esta se torne organofílica, isto é, compatível com polímeros orgânicos ${ }^{[3,4]}$.

Os nanocompósitos podem ser produzidos por polimerização in situ, intercalação por solução e intercalação por fusão. Esta última tem sido bastante utilizada devido ao material ser processado em equipamentos de misturas convencionais, não necessitando assim do uso de solventes, o que é interessante do ponto de vista ambiental. As estruturas que podem ser obtidas do híbrido polímero/argila são: compósito convencional e nanocompósitos intercalado, esfoliado ordenado e esfoliado desordenado ${ }^{[4-9]}$.

A partir das pesquisas realizadas pelo grupo da Toyota que desenvolveu nanocompósitos de poliamida 6 (PA6) com pequenas quantidades de argila montmorilonita e melhoramento significativo das propriedades mecânicas e térmicas do nanocompósito obtido, vários outros estudos têm sido realizados a fim de se obter uma sinergia entre os componentes, dada à importância do desenvolvimento desse tipo de material ${ }^{[4-20]}$. As diversas pesquisas têm estudado vários fatores que podem contribuir para o melhoramento das propriedades do nanocompósito, entre eles podem se destacar: o peso molecular da matriz polimérica, o tipo de sal orgânico, o teor de argila, condições operacionais dos equipamentos de processamento, entre outros ${ }^{[6-9,17,22]}$. Este trabalho tem como objetivo analisar a influência do peso molecular da poliamida 6 nas propriedades morfológicas, mecânicas e termomecânicas de nanocompósitos de PA6/argila organofílica.

\section{Experimental}

\section{Material}

A matriz empregada foi a poliamida 6 da Rhodia/ SP, com três pesos moleculares: baixo $(\mathrm{BPM})=$ 7.100 g. $\mathrm{mol}^{-1}$, IV $=120$ mL.g ${ }^{-1}$ (Technyl 97519); médio $(\mathrm{MPM})=10.500 \mathrm{~g} \cdot \mathrm{mol}^{-1}, \mathrm{IV}=134 \mathrm{~mL} \cdot \mathrm{g}^{-1}$ (Technyl C216); e alto $(\mathrm{APM})=18.800 \mathrm{~g} \cdot \mathrm{mol}^{-1}, \mathrm{IV}=225 \mathrm{~mL} \cdot \mathrm{g}^{-1}$ (Technyl B400), conforme dados fornecidos pelo fabricante, sob a forma de grânulos de coloração branca. O polímero foi usado como recebido. A argila BRASGEL PA (sódica ativada), de cor creme, fornecida pela Indústria Bentonit União Nordeste (BUN), Campina Grande - PB, foi utilizada após passagem em peneira ABNT $\mathrm{n}^{\circ} 200(\mathrm{~d}=0,074 \mathrm{~mm})$. A capacidade de troca de cátions (CTC) dessa argila é de 90 meq. $100 \mathrm{~g}^{-1}$ (método de adsorção de azul de metileno). Para tornar a argila compatível com o polímero, os íons de sódio presentes entre as lamelas da argila são trocados por íons de amônio para produzir a argila organofílica. Essa troca foi realizada na presença do sal quaternário de amônio Genamin (cloreto de hexadeciltrimetil amônio), fornecido por Clariant/PE. Para a obtenção da argila organofílica (OMMT) foi realizado tratamento apropriado para o sal Genamin, baseando-se na CTC da argila, conforme trabalhos já reportados pelo $\operatorname{grupo}^{[3,11,15,18,21]}$.

\section{Métodos}

\section{Caracterização da argila}

A análise por Fluorescência de raio $\mathrm{X}$ da argila foi realizada pelo método semiquantitativo, em forma de pastilha, sob atmosfera de He, em equipamento modelo EDX 700 da Shimadzu, pertencente ao Laboratório do Centro de Tecnologia do Gás-CTGás, Natal-RN.

As análises de DRX foram realizadas em aparelho XRD-6000 Shimadzu, utilizando-se radiação K $\alpha$ do cobre, tensão de $40 \mathrm{kV}$, corrente de $30 \mathrm{~mA}$, varredura entre $2 \theta$ de 2 a $30^{\circ}$ e velocidade de varredura de $2 \%$ min, no Laboratório de Engenharia de Materiais/CCT/UFCG.

\section{Preparação dos nanocompósitos}

Para a obtenção dos nanocompósitos de poliamida 6 com três pesos moleculares e argila, inicialmente, foram preparados concentrados $(50 \%$ de poliamida 6 e $50 \%$ de argila, ou seja 1:1) em misturador interno acoplado ao Reômetro de Torque System 90 da Haake-Büchler, operando a $240{ }^{\circ} \mathrm{C}$, $60 \mathrm{rpm}$ por 10 minutos. Os concentrados obtidos foram triturados em moinho de facas e, posteriormente, adicionados à matriz polimérica, em quantidades necessárias para a obtenção de teores nominais de $3 \%$ em massa de argila, visto que em pesquisas realizadas anteriormente pelo nosso grupo de estudos, ficou evidenciado que os nanocompósitos obtidos apresentaram melhores propriedades com este teor ${ }^{[4,11,15,18-21,23]}$. As misturas foram processadas em extrusora de rosca dupla contra-rotativa acoplada ao reômetro, utilizando temperatura de $230{ }^{\circ} \mathrm{C}$ na $1^{\mathrm{a}}$ zona e $240{ }^{\circ} \mathrm{C}$ nas demais zonas de aquecimento e velocidade de rotação das roscas de $60 \mathrm{rpm}$. Para efeito de comparação, as poliamidas puras com os três pesos moleculares foram extrusadas sob as mesmas condições das misturas.

Todos os materiais à base de poliamida 6 foram secados a $80 \pm 5{ }^{\circ} \mathrm{C}$ por 24 horas em estufa a vácuo, para a retirada de umidade, antes de serem processados. As misturas obtidas foram trituradas em moinho de facas e injetadas em equipamento Fluidmec a $240{ }^{\circ} \mathrm{C}$, na forma de corpos de prova de 
tração (ASTM D638) e impacto (ASTM D256). As temperaturas do molde foram diferenciadas de acordo com o peso molecular da matriz para favorecer o preenchimento do mesmo, visto que a poliamida 6 de alto peso molecular não preenchia o molde quando este se encontrava a menos de $80^{\circ} \mathrm{C}$. A temperatura do molde foi de $80{ }^{\circ} \mathrm{C}$ para a poliamida de alto peso molecular e de $40{ }^{\circ} \mathrm{C}$ para a poliamida de baixo e médio pesos moleculares. $\mathrm{O}$ tempo de molde fechado foi de 20 segundos.

\section{Caracterização dos nanocompósitos}

Para a avaliação da formação dos nanocompósitos de poliamida 6 com diferentes pesos moleculares/argila organofílica, os sistemas foram caracterizados por DRX, pois esta técnica possibilita determinar o espaçamento basal $\left(\mathrm{d}_{001}\right)$ entre as camadas da argila. As análises de DRX nos corpos de prova foram feitas em aparelho XRD-6000 Shimadzu, utilizando-se radiação K $\alpha$ do cobre, tensão de $40 \mathrm{kV}$, corrente de $30 \mathrm{~mA}$, varredura entre $2 \theta$ de 2 a $30^{\circ}$ e velocidade de varredura de $2 \%$ min, no Laboratório de Engenharia de Materiais/ CCT/UFCG.

A microscopia eletrônica de transmissão permite analisar localmente a morfologia formada nos nanocompósitos e, portanto, fornece uma representação melhor de sua estrutura. As análises de MET foram realizadas utilizando-se o microscópio eletrônico de transmissão PHILIPS CM120, operando a uma voltagem de aceleração de $120 \mathrm{kV}$, pertencente ao DEMA/UFSCar. As amostras analisadas foram retiradas do centro do corpo de prova de impacto. Foram preparadas através da redução de área pelo procedimento de "trimmer", em forma trapezoidal com uma área de aproximadamente $0,5 \mathrm{~mm}^{2}$. Os cortes das amostras foram realizados em um ultramicrótomo RMC modelo MT-7000 usando-se uma faca de diamante da marca Diatome tipo Cryohisto $45^{\circ}$, em condições criogênicas, com velocidade de corte de $0,1 \mathrm{~mm} / \mathrm{s}$ e espessura entre 25 a $50 \mathrm{~nm}$.

A caracterização mecânica sob tração foi realizada segundo a norma ASTM D 638 em máquina universal de ensaios LLoyd LR-10KN, com velocidade de deformação de 5mm/min, no Laboratório de Caracterização de Materiais/ CCT/UFCG. Foram ensaiados no mínimo 10 corpos de prova para cada composição.

A análise térmica dinâmico-mecânica (DMTA) mede a resposta de um determinado material a uma deformação oscilatória (neste caso, modo de tensão-torção) como função da temperatura. Os ensaios foram realizados nos corpos de prova de impacto, sem entalhe, no modo de flexão em três pontos, com frequiência de $1 \mathrm{~Hz}$ e amplitude de deformação de $0,1 \%$, em um equipamento da TA Instruments, na faixa de temperatura de 25 a $200{ }^{\circ} \mathrm{C}$, em atmosfera de ar, pertencente ao DEMat/CT/UFRN.

A temperatura de deflexão térmica (HDT) foi obtida, conforme a norma ASTM D 648, em um equipamento Ceast, modelo HDT 6 VICAT P/N 6921.000, pertencente ao $\mathrm{DEMa} / \mathrm{UFSCar}$, com uma tensão de 1,82 MPa, taxa de aque- cimento de $120^{\circ} \mathrm{C} / \mathrm{h}$ (método A), onde o meio de imersão foi um óleo de silicone. A temperatura foi determinada após a amostra ter defletido 0,25 $\mathrm{mm}$. Uma série de cinco amostras foi ensaiada e a temperatura de deflexão térmica, com seu respectivo desvio-padrão, reportados.

\section{Resultados e Discussão}

\section{Caracterização da argila}

Na Tabela 1, observa-se uma composição elementar característica das argilas. Para a argila sem tratamento (MMT), pode-se destacar a presença de óxido de sódio $\left(\mathrm{Na}_{2} \mathrm{O}\right)$ e traços de cloro. Para a argila organofílica (OMMT), o sódio não está mais presente e a presença do cloro é mais pronunciada. Estes resultados podem indicar que ocorreu a troca de cátions nos espaços interlamelares da argila, tornando-a organofílica, conforme também atestado por Paz e Barbosa ${ }^{[4,15]}$.

\section{Caracterização dos nanocompósitos}

\section{Difração de raio $X$ (DRX)}

A Figura 1 mostra os difratogramas de DRX da argila sem tratamento (MMT), da argila organofílica (OMMT) e dos corpos de prova de impacto dos sistemas de poliamida com diferentes pesos moleculares/argila organofílica. Comparando-se os difratogramas, pode-se visualizar que houve um deslocamento do pico do ângulo $2 \theta$ de $7,3^{\circ}$ (MMT) para 4,06 (OMMT), ou seja, a distância basal, $\mathrm{d}_{001}$, passou de 12,1 Å para $21,7 \AA$, respectivamente. Isto indica que houve a intercalação das moléculas do sal entre as camadas da argila, conforme re-

Tabela 1. Composição química da argila sem tratamento (MMT) e da argila organofílica (OMMT) obtida por meio da técnica de Fluorescência de raio $X$.

\begin{tabular}{ccc}
\hline Óxidos & MMT $(\%)$ & OMMT $(\%)$ \\
\hline $\mathrm{SiO}_{2}$ & 62,85 & 63,55 \\
$\mathrm{Al}_{2} \mathrm{O} 3$ & 18,02 & 18,66 \\
$\mathrm{Fe}_{2} \mathrm{O} 3$ & 11,25 & 11,91 \\
$\mathrm{MgO}$ & 2,06 & 1,60 \\
$\mathrm{CaO}$ & 1,78 & 0,96 \\
$\mathrm{TiO}_{2}$ & 1,22 & 1,28 \\
$\mathrm{Na}_{2} \mathrm{O}$ & 1,12 & - \\
$\mathrm{K}_{2} \mathrm{O}$ & 0,85 & 0,84 \\
$\mathrm{Cl}$ & 0,41 & 0,76 \\
$\mathrm{SO}_{3}$ & 0,09 & 0,10 \\
$\mathrm{MnO}_{2}$ & 0,08 & 0,04 \\
$\mathrm{P}_{2} \mathrm{O}_{5}$ & 0,07 & 0,06 \\
$\mathrm{Cr}_{2} \mathrm{O}_{3}$ & 0,07 & 0,07 \\
$\mathrm{CuO}_{\mathrm{ZnO}}$ & 0,05 & 0,05 \\
$\mathrm{SrO}^{\mathrm{ZrO}}$ & 0,03 & 0,04 \\
$\mathrm{NiO}$ & 0,03 & 0,03 \\
& 0,02 & 0,02 \\
\hline
\end{tabular}




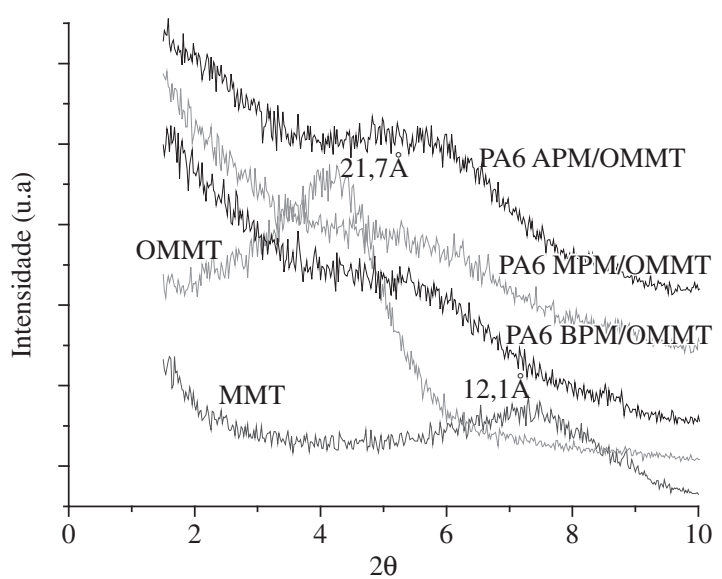

Figura 1. Difratogramas da argila sem tratamento (MMT), da argila organofílica (OMMT) e dos nanocompósitos de: PA6 BPM/OMMT, PA6 MPM/OMMT e PA6 APM/OMMT.

latado em outros trabalhos ${ }^{[15,18-21]}$ que também utilizaram este sal quaternário de amônio na organofilização de argilas bentonitas de mesma procedência. Observa-se também um ombro em todos os sistemas de PA6/OMMT, entre o espaçamento basal da MMT $\left(d_{001}=12,1 \AA\right)$ e da OMMT $\left(d_{001}=21,7 \AA\right)$. Esses resultados indicam que os nanocompósitos apresentaram morfologias com partículas parcialmente esfoliadas e intercaladas pelo polímero, o que será confirmado por meio da microscopia eletrônica de transmissão. Uma explicação possível para tal observação é que pode ter ocorrido degradação parcial do sal e, por esta razão, a distância $\mathrm{d}_{001}$ ter sido reduzida em comparação com a argila organofílica (OMMT). Segundo Zanetti e Costa $^{[24]}$, a presença do ombro pode indicar um aumento da distância basal devido à intercalação do polímero.

\section{Microscopia eletrônica de transmissão (MET)}

As Figuras 2a, 2b, 2c mostram as fotomicrografias dos sistemas de poliamida 6 com $3 \%$ em peso de argila organofílica. A fotomicrografia do sistema PA6 BPM/OMMT (Figura 2a) mostra uma morfologia parcialmente esfoliada composta de lamelas de argila bem distribuídas na matriz polimérica, com áreas contendo lamelas esfoliadas e pequenas áreas com estruturas intercaladas. O sistema PA6 MPM/OMMT (Figura 2b) apresenta também morfologia parcialmente composta de lamelas e alguns aglomerados de argilas dispersos na matriz. Já o sistema PA6 APM/OMMT (Figura 2c) exibe uma morfologia composta predominantemente de aglomerados de lamelas de argila na matriz polimérica. Estes resultados são contraditórios aos que foram relatados por Fornes et al. ${ }^{[7]}$, que afirmaram que o alto peso molecular do polímero e, portanto, sua maior viscosidade contribui para uma maior energia para separar as lamelas da argila, enquanto a matriz de menor peso molecular favorece à formação de aglomerados. Uma possível explicação para os resultados obtidos é que quanto menor o peso molecular do polímero mais favorável será o processo de difusão das moléculas para dentro das galerias da argila, o que pode ter ocorrido com os polímeros de BPM e MPM. Já com a ma-

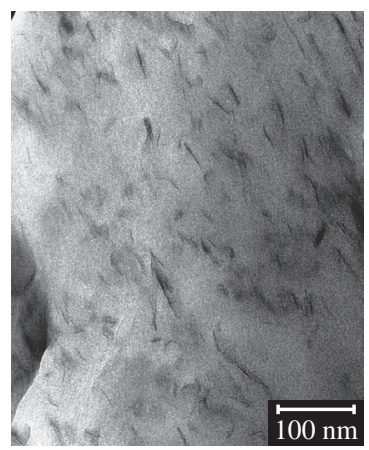

(a)

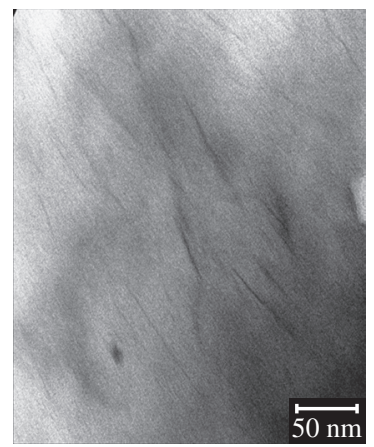

(b)

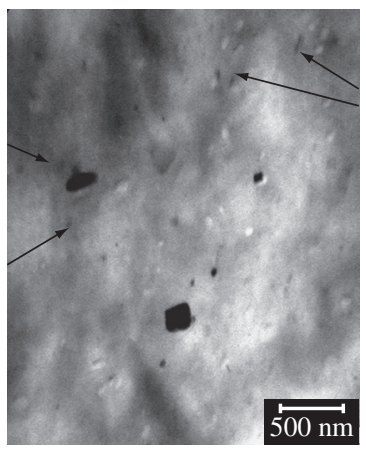

(c)

Figura 2. Fotomicrografias de MET das amostras dos nanocompósitos de: a) PA 6 BPM/OMMT; b) PA 6 MPM/OMMT; e c) PA 6 APM/OMMT.

triz de APM, a presença de aglomerados, mostrados pelas setas, pode ser atribuída à maior viscosidade do polímero. Além disso, o equipamento de mistura utilizado e suas condições operacionais podem não terem sido suficientes para promover o cisalhamento adequado para delaminação/esfoliação da argila. Resultados semelhantes foram observados e mencionados por Meng et al. ${ }^{[22]}$. Embora a microscopia eletrônica de transmissão tenha revelado que os polímeros com BPM e MPM tenham morfologias com partículas mais esfoliadas, a difração de raio $\mathrm{X}$ não mostrou diferenças significativas entre os polímeros de baixo, médio ou alto peso molecular. Isto confirma que o uso combinado destas duas técnicas é importante na interpretação do tipo de nanocompósito formado.

\section{Caracterização mecânica sob tração}

A Tabela 2 apresenta as propriedades mecânicas sob tração obtidas para a poliamida 6 e seus nanocompósitos. Percebe-se que já existe uma diferença, embora pequena, no valor do módulo sob tração para os componentes puros, ou seja, este valor é maior para a poliamida 6 de médio peso molecular e, conseqüentemente, se mantem superior no nanocompósito obtido com esta matriz. Entretanto, observa-se que os sistemas de poliamida 6/OMMT, em geral, exibiram melhores propriedades mecânicas sob tração quando comparadas às propriedades da poliamida 6 , ou seja, a argila atuou como uma carga reforçante aumentando a rigidez e a resistência do sistema, como pode ser verificado por meio do módulo de 
Tabela 2. Propriedades mecânicas da poliamida 6 com diferentes pesos moleculares e seus nanocompósitos.

\begin{tabular}{cccc}
\hline Material & $\begin{array}{c}\text { Módulo de } \\
\text { elasticidade } \\
(\mathbf{G P a})\end{array}$ & $\begin{array}{c}\text { Tensão no } \\
\text { escoamento } \\
(\mathbf{M P a})\end{array}$ & $\begin{array}{c}\text { Alongamento } \\
\text { no escoamento } \\
(\%)\end{array}$ \\
\hline PA6 BPM & $1,67 \pm 0,1$ & $60,10 \pm 1,6$ & $4,85 \pm 0,7$ \\
PA6 MPM & $1,78 \pm 0,1$ & $54,55 \pm 2,0$ & $4,69 \pm 0,4$ \\
PA6 APM & $1,73 \pm 0,1$ & $64,44 \pm 4,0$ & $4,62 \pm 0,4$ \\
PA6 & $1,83 \pm 0,0$ & $63,19 \pm 1,1$ & $4,99 \pm 0,6$ \\
BPM/OMMT & & & \\
PA6 & $2,27 \pm 0,2$ & $64,26 \pm 2,6$ & $4,35 \pm 0,2$ \\
MPM/OMMT & & & \\
PA6 & $2,33 \pm 0,1$ & $69,32 \pm 0,3$ & $4,12 \pm 0,1$ \\
APM/OMMT & & & \\
\hline
\end{tabular}

elasticidade e da tensão no escoamento. Estes resultados são similares aos encontrados por Chiu et al. ${ }^{[17]}$ e Souza ${ }^{[23]}$.

\section{Análise térmica dinâmico-mecânica (DMTA)}

As Figuras 3a, 3b, 3c mostram os resultados obtidos por DMTA para as poliamidas com diferentes pesos moleculares e seus nanocompósitos. A incorporação da argila organofílica, OMMT, nas matrizes poliméricas de poliamida 6, aumentou significativamente a rigidez do sistema (E'-módulo de armazenamento), principalmente para os nanocompósitos com baixo peso molecular e, diminuiu o $\tan \delta$, para todas as temperaturas estudadas, confirmando o efeito reforçante da argila no polímero. Este comportamento indica melhoramento da estabilidade termo-mecânica destes materiais em temperaturas altas. É interessante perceber que o valor do módulo de rigidez se destaca para
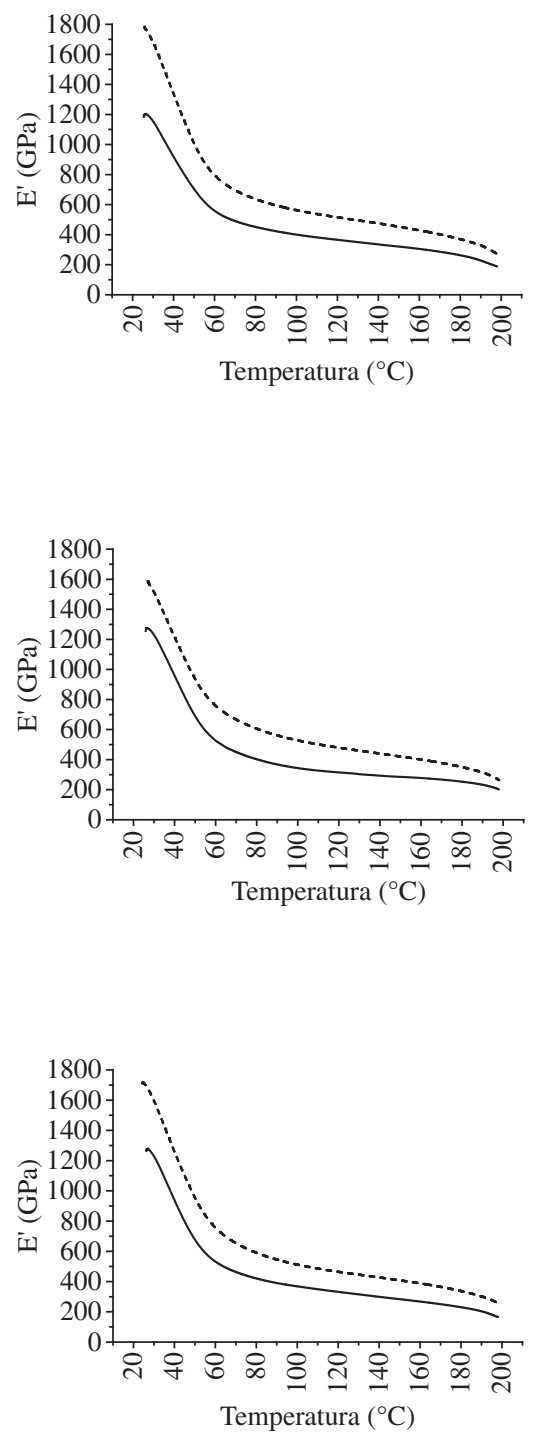

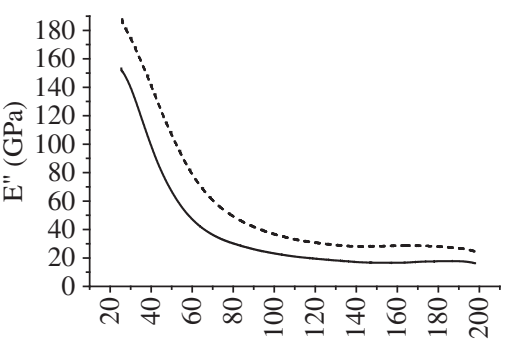

Temperatura $\left({ }^{\circ} \mathrm{C}\right)$

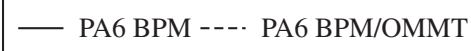

(a)

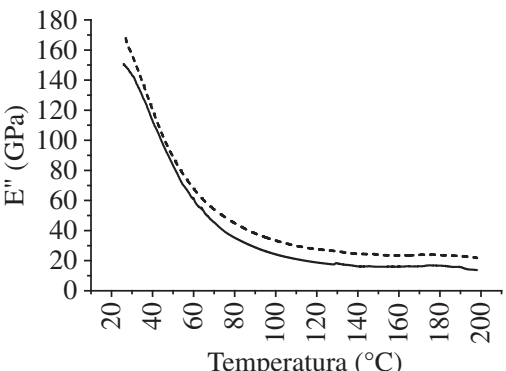

Temperatura $\left({ }^{\circ} \mathrm{C}\right)$

— PA6 MPM - - - PA6 MPM/OMMT

(b)

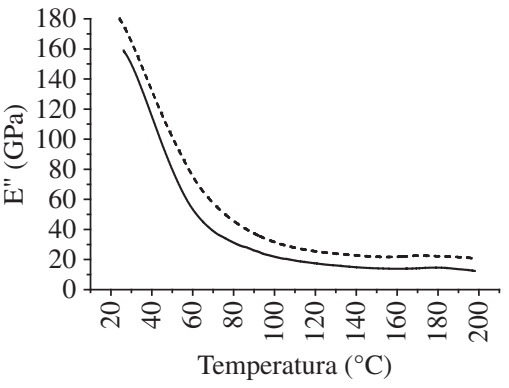

PA6 APM - - - PA6 APM/OMMT

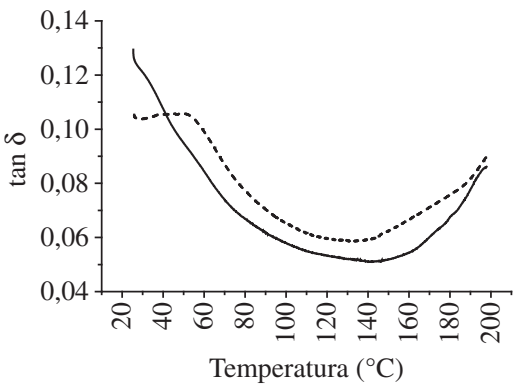

Temperatura $\left({ }^{\circ} \mathrm{C}\right)$

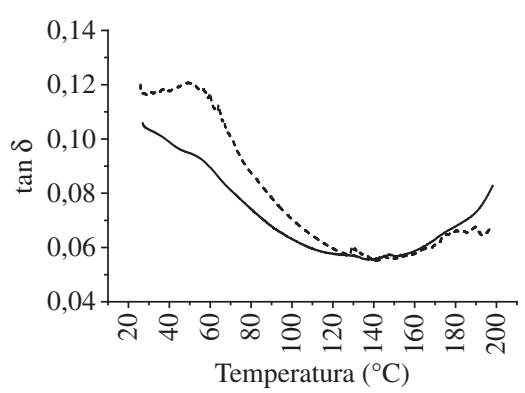

(c)

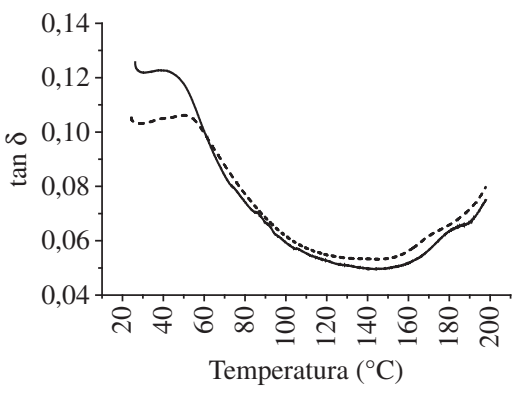

Figura 3. Curvas de DMTA para a poliamida 6 com diferentes pesos moleculares e seus nanocompósitos: a) PA6 BPM/OMMT; b) PA6 MPM/OMMT; e c) PA6 APM/OMMT. E' (módulo de armazenamento), E” (módulo de perda) e tan $\delta$ em função da temperatura. 
o material de menor viscosidade provavelmente por que a dispersão das lamelas de argila foi mais eficiente nesta matriz do que na de alto peso molecular e, em função disso, tem-se maior reforçamento do silicato. A principal razão para o melhoramento dessa propriedade nos nanocompósitos pode estar relacionada com a interação interfacial mais forte entre a matriz e o silicato em camada, devido à alta razão de aspecto das lamelas da argila, comparada aos sistemas convencionais reforçados com carga, conforme reportado por Sinhá Ray e Okamoto ${ }^{[9]}$. Observa-se ainda nitidamente, por meio das curvas de $\tan \delta$, a Tg da poliamida em torno de $50{ }^{\circ} \mathrm{C}$.

\section{Temperatura de deflexão térmica (HDT)}

A Figura 4 e a Tabela 3 mostram os dados obtidos para a temperatura de deflexão térmica da poliamida 6 com diferentes pesos moleculares e seus nanocompósitos. Em geral, a HDT da poliamida 6 ficou na faixa de $49{ }^{\circ} \mathrm{C}$ em média e à dos nanocompósitos acima de $55^{\circ} \mathrm{C}$. Apesar de ser um aumento modesto, implica numa melhoria dessa propriedade, o que é importante do ponto de vista de aplicação. Este aumento pode ser atribuído à ocorrência da dispersão das camadas do silicato na matriz polimérica, conforme reportado na literatura ${ }^{[9,23]}$, o que eleva as propriedades mecânicas e termomecânicas.

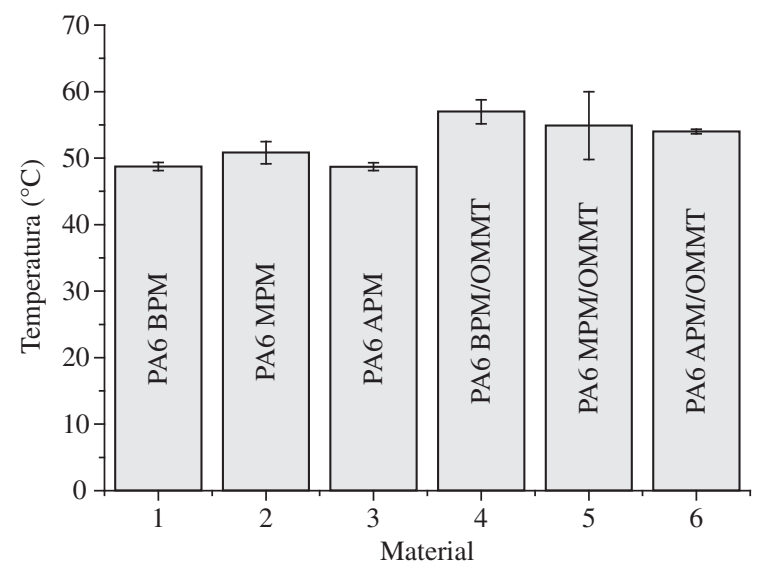

Figura 4. Temperatura de deflexão térmica (HDT) da poliamida 6 com diferentes pesos moleculares e seus nanocompósitos: PA6 BPM/OMMT, PA6 MPM/OMMT e PA6 APM/OMMT.

Tabela 3. Temperatura de deflexão térmica da poliamida 6 com diferentes pesos moleculares e seus nanocompósitos.

\begin{tabular}{cc}
\hline Material & HDT $\left({ }^{\circ} \mathbf{C}\right)$ \\
\hline PA6 BPM & $48,70 \pm 0,6$ \\
PA6 MPM & $50,80 \pm 1,7$ \\
PA6 APM & $48,67 \pm 0,5$ \\
PA6 BPM/OMMT & $56,97 \pm 1,8$ \\
PA6 MPM/OMMT & $54,87 \pm 5,1$ \\
PA6 APM/OMMT & $54,00 \pm 0,3$ \\
\hline
\end{tabular}

\section{Conclusões}

Nanocompósitos de poliamida 6 com três pesos moleculares e argila organofílica foram obtidos por intercalação por fusão. Por meio das técnicas de difração de raio X e fluorescência de raio $\mathrm{X}$ foi confirmada a presença das moléculas do sal quaternário de amônio na argila e, sua organofilização. Os difratogramas de raio $\mathrm{X}$ e a morfologia obtida por MET revelaram que os nanocompósitos de poliamida 6 de baixo e médio pesos moleculares exibiram estrutura esfoliada e/ou parcialmente esfoliada. Já os nanocompósitos com matriz de alto peso molecular apresentaram também presença de aglomerados. Os resultados obtidos por DMTA e HDT confirmaram o efeito reforçante da argila no polímero por meio do aumento da rigidez (E') do sistema e da temperatura de deflexão térmica, principalmente para o nanocompósito com poliamida 6 de baixo peso molecular. Enquanto que o módulo de elasticidade obtido sob tração foi maior para o sistema de poliamida $6 \mathrm{com}$ alto peso molecular.

\section{Agradecimentos}

Os autores agradecem à Rhodia/SP pela doação da poliamida 6, à Bentonit União Nordeste pela doação da argila, à Clariant/PE pela doação do sal, ao LABMat - Laboratório de Eng. de Materiais/CCT/UFCG, ao Prof. Dr. José Daniel D. Melo (DEMat/UFRN) pela atenção e colaboração na realização dos ensaios de DMTA, ao MCT/CNPq e à Rede de Nanotecnologia Molecular e de Interfaces (RENAMI) pelo auxílio financeiro.

\section{Referências Bibliográficas}

1. Leite, I. F. - "Avaliação das propriedades de duas diferentes bentonitas para uso como cargas em nanocompósitos poliméricos", in: Anais do $50^{\circ}$ Congresso Brasileiro de Cerâmica, p.1, Blumenau-SC, maio (2006).

2. Ganguli, S.; Dean, D.; Jordan, K.; Price, G. \& Vaia, R. Polymer, 44, p.1315, (2003).

3. Barbosa, R.; Araújo, E. M.; Oliveira, A. D. \& Melo, T. J. A. - Revista Cerâmica, 52, p. 264, (2006).

4. Paz, R. A. - "Efeito do peso molecular da poliamida 6 no desenvolvimento de nanocompósitos", Dissertação de Mestrado, Universidade Federal de Campina Grande, Brasil (2008).

5. Vaia, R. A.; Ishii, H. \& Giannelis, E. P. - Chemistry of Materials, 5, p.1694, (1993).

6. Alexandre, M.; Dubois, P. - Mat. Sci. and Eng., 28, p.1, (2000).

7. Fornes, T. D.; Yoon, P. J.; Keskkula, H. \& Paul, D. R. - Polymer, 42, p.9929, (2001).

8. Wang, K. H.; Choi, M. H.; Koo, C. M.; Choi, Y. S. \& Chung, I. J. - Polymer, 42, p.9819, (2001). 
9. Sinha Ray, S. \& Okamoto M. - Progress in Pol. Sci., 28, p.1539, (2003).

10. Araújo, S. S.; Paiva, G. P.; Carvalho, L. H. \& Silva, S. M. L. - Revista Matéria, 9, (4), p. 426, (2004).

11. Araújo, E. M.; Melo, T. J. A.; Santana, L. N. L.; Neves, G. A.; Ferreira, H. C.; Lira, H. L.; Carvalho, L. H.; Ávila Jr., M. M.; Pontes, M. K. G. \& Araújo, I. S. Mat. Sci. and Eng. B, 112, p.175, (2004).

12. Esteves, Ana C. C.; Timmons A. B. \& Trindade, T. - Química Nova, 27, p.798, (2004).

13. Pedroni, L. G.; Oviedo, M. A. S.; Rosolen, J. M. \& Nogueira, A. F. - "Preparação e caracterização de nanocompóstios poliméricos de MWCNT / Kraton-D", in: 29 ${ }^{a}$ Reunião Anual da Sociedade Brasileira de Química, São Paulo, (2004).

14. Yuan, M.; Turng, L. S.; Gong, S. \& Winardi, A. - Journal of Cellular Plastics, 40, p. 397, (2004).

15. Barbosa, R. - "Efeito de sais quaternários de amônio na organofilização de uma argila bentonita nacional para o desenvolvimento de nanocompósitos de polietileno de alta densidade (PEAD)”, Dissertação de Mestrado, Universidade Federal de Campina Grande, Paraíba, Brasil (2005).

16. Filho, F. G. R.; Junior, M. M. A.; Santana, L. L.; Melo, T. J. A. \& Silva, S. M. L. - Revista Matéria, 10, p.24, (2005).
17. Chiu, F. C.; Lai, S. M.; Chen, Y. L. \& Lee T. H. - Polymer, 46, p.11600, (2005).

18. Araújo, E. M.; Melo, T. J. A.; Oliveira, A D.; Araújo, H. L. D.; Araújo, K. D. \& Barbosa, R. - Polimeros: Cienc Tecnol, 16, p.38, (2006).

19. Araújo, E. M.; Barbosa, R.; Oliveira, A. D.; Morais, C. R. S.; Mélo, T. J. A. \& Souza, A. G. - J. of Therm. Analy. and Cal., 87, (3), p.811, (2007).

20. Araújo, E. M.; Barbosa, R.; Morais, C. R. S.; Soledade, L. E. B.; Souza, A. G. \& Vieira, M. Q. - J. of Therm. Analy. and Cal., 90, p.841, (2007).

21. Araújo, E. M.; Barbosa, R.; Rodrigues, A. W. B. \& Ito, E. N. - Mat. Sci. and Eng. A, 445-446, p.141, (2007).

22. Meng, X.; Wang, Z.; Zhao, Z.; Du, X.; Bi, W. \& Tang, T. - Polymer, 48, p.2508, (2007).

23. Souza, M. A. - "Obtenção de nanocompósitos de poliamida 6/argila nacional com diferentes agentes compatibilizantes", Dissertação de Mestrado, Universidade Federal de São Carlos, São Paulo, Brasil (2006).

24. Zanetti, M. \& Costa, L. - Polymer, 45, p.4367, (2004).

Enviado: $14 / 04 / 08$

Reenviado: 11/06/08

Aceito: $23 / 06 / 08$ 